\title{
EDITORIAL
}

\section{Special Issue on Tribology of Advanced Materials}

\author{
Alison C. Dunn ${ }^{1} \cdot$ Brandon A. Krick $^{2} \cdot$ Kenneth M. Liechti ${ }^{3} \cdot$ Frank W. DelRio $^{4}$
}

Published online: 21 October 2021

(c) Society for Experimental Mechanics 2021

The word tribology is derived from the Greek word tribos meaning "rubbing" and the suffix ology meaning "the study of", and thus represents the study of interacting surfaces in relative motion. More specifically, tribology focuses on the science and engineering of three main phenomena: friction, wear, and lubrication. These phenomena are ubiquitous in most mechanical components and manufacturing processes, and play a key role in the energy consumption and economic expenditure of the transport, manufacturing, power generation, and residential sectors. This special issue aims to exchange recent progress in the field of tribology, with an emphasis on the development and application of new experimental mechanics tools to address fundamental and applied problems at all length and time scales. The issue will not only describe work on classic problems such as hard-material adhesion and friction, but will also showcase work in new disciplines such as micro- and nanotechnology, and biology and medicine. In addition, the issue will highlight the interdisciplinary nature of the field, with contributions that leverage physics, chemistry, biology, mathematics, and engineering to address complex problems with advanced materials. In total, the collection consists of nine papers: one review paper and eight full-length articles. The issue starts with the review paper and subsequently moves to the eight full-length articles, the latter of which are grouped into two categories based on the predominant focus, namely elastic and viscoelastic properties and friction and contact mechanics.

The review paper describes recent work related to the lubrication mechanisms of ionic lubricants (ILs), which have attracted considerable attention due to their unique

Frank W. DelRio

fwdelri@sandia.gov

1 University of Illinois Urbana-Champaign, Urbana, IL 61801, USA

2 Florida State University, Tallahassee, FL 32306, USA

3 University of Texas At Austin, Austin, TX 78712, USA

4 Sandia National Laboratories, Albuquerque, NM 87185, USA physicochemical properties and promising lubrication performance when used in diverse material pairs. In Recent advances in nanotribology of ionic liquids, $\mathrm{Li}$ and Mangolini summarize and analyze recent state-of-the-art experiments on the effects of normal pressure, nanoconfinement, roughness, charge density, and impurities on the lubrication mechanisms and properties of ILs. In addition, the authors highlight the growing interest in exploring the pathways and kinetics of stress-assisted, thermally-activated tribofilms occurring at these interfaces; to this end, several groups have developed novel atomic force microscopy (AFM)-based methods for in-situ observations of tribofilm growth kinetics as a function of normal pressure, contact cycles, and temperature. Finally, the authors identify several open questions in the field, the most prominent being the effects of temperature, residual halides from the synthesis process, roughness, and crystallographic alignment on the friction response of ILs. A foreseeable pathway to addressing these questions includes the continued development of in-situ experimental tools with advanced spectroscopic and imaging methods and computational tools to enable a holistic molecular-scale understanding of the phenomena controlling the friction response.

The section on elastic and viscoelastic properties includes four papers on the deformation, strain, elastic modulus, and load-relaxation behavior of soft materials, with the idea that these properties dictate the contact area and ultimately the tribological behavior. In Bending of soft micropatterns in elastohydrodynamic lubrication tribology, Peng et al. investigate and model the differences in lubricated friction for poly(dimethyl siloxane) (PDMS) elastomer and poly(ethylene glycol) diacrylate (PEGDA)/alginate double network (DN) hydrogel micropatterns to assess the effect of height-towidth aspect ratio and bending angle on elastohydrodynamic friction. The authors note that the PDMS-PDMS tribopairs exhibit a friction peak that is independent of aspect ratio for short patterns, but demonstrate a "power-law fit" decrease with increasing aspect ratio for taller patterns. The decrease in lubricated friction is attributed to taller patterns having large bending angles and a reduced effective surface for fluid load bearing. In Dynamic subsurface deformation and strain of 
soft hydrogel interfaces using an embedded speckle pattern with $2 D$ digital image correlation, McGhee et al. develop a new digital image correlation (DIC) method to acquire fullfield subsurface deformation of hydrogels in real time. The method is based on a speckle pattern that is deposited through the central portion of the sample and polymerized using photoinitiation to lock the pattern in place. The authors utilize the approach to measure subsurface deformation and strain fields associated with indentation, relaxation, and sliding experiments in a hydrogel. In Load-relaxation characteristics of chemical and physical hydrogels as soft tissue mimics, Islam and Oyen quantitatively characterize the load-relaxation behavior of physical (agar, alginate, and gelatin) and chemical (polyacrylamide) hydrogels and perform a comparative analysis to several biological tissues. The physical gels exhibit strong time-dependent behavior (i.e., load-relaxation is dominated by viscoelasticity), while the chemical gel shows negligible relaxation in short time scales (i.e., load-relaxation is controlled by poroelasticity). The comparative analysis reveals that physical gels are more representative of biological tissues in terms of time-dependent properties, whereas chemical hydrogels better mimic the porous structure of soft tissue. In Microindentation of cartilage before and after articular loading in a bioreactor: assessment of length-scale dependency using two analysis methods, Yuh et al. use two different methods (Oliver-Pharr and Hertzian contact mechanics models) to determine the reduced elastic moduli of articular cartilage at varying length-scales (tip radii from $20 \mu \mathrm{m}$ to $793.75 \mu \mathrm{m}$ ) before and after bioreactor loading. The authors show that the reduced moduli from Hertzian mechanics were similar for all tip radii, but also reveal a deviation in moduli values at larger radii when utilizing the Oliver-Pharr model due to adhesion.

The section on friction and contact mechanics includes two papers on speed-dependent and time-dependent frictional behavior of hydrogel surfaces and two papers on contact mechanics models for soft and hard interfaces. In Tribological characterization of gradient-density polyacrylamide hydrogel surfaces, Johnson and Dunn examine the tribological response of a "brushy" less-dense polymer film on a hydrogel surface via indentation, creep, and sliding experiments as function of load, speed, and probe material. The authors reveal a non-Hertzian regime for the first $13 \mu \mathrm{m}$ to $29 \mu \mathrm{m}$ after initial contact, poroelastic relaxation with water exudation in the "brushy" less-dense film, and highly speed-dependent friction with larger speeds translating to friction coefficients as small as 0.01 . The absence of transient behavior is thought to be indicative of quick water uptake by the surface film immediately after each experiment. In Relaxation and recovery in hydrogel friction on smooth surfaces, Wu et al. survey the time-dependent frictional behavior of hydrogels over multiple time scales and show that the behavior is consistent with sliding-induced relaxation and quiescent recovery. At a fixed velocity, the authors also illustrate that the friction coefficient decreases exponentially to a steady-state value. The recovery (increase) in friction after shearing suggests that the hydrogel surface has been structurally altered at higher velocities. Finally, it is shown that the initial direction of shear imprints a "memory" that can be observed at long times; the authors surmise that this memory is due to some semi-permanent orientational ordering at the interface. In A simple contact mechanics model for highly strained aqueous surface gels, Chau et al. develop a model to predict the force response of a probe indenting compositionally-graded materials with soft surface layers under high strains and deformations. The model takes concepts from polymer physics, fluid draining, and Winkler foundation mechanics and uses them to relate applied force to probe radius, elastic modulus, and film thickness. The authors evaluate the model on exemplar graded surface gels (contact lenses and stratified hydrogels) and show that the model captures the nonlinear contact mechanics of the highly-strained materials better than the Hertzian or Winkler models. In Hard-material adhesion: Which scales of roughness matter most?, Thimons et al. aim to determine which scales of roughness have the strongest effect on macroscopic adhesion, using diamond coatings with varying roughnesses and spherical ruby probes large enough to sample all length scales. The intrinsic work of adhesion and range of adhesion for ruby and polycrystalline diamond are found to be $46.3 \mathrm{~mJ} / \mathrm{m}^{2}$ and $5.6 \mathrm{~nm}$, respectively, using topography and adhesion data and numerical integration of an interaction potential. The large adhesion range and plasticity at the interface suggest a diminished role of small-scale topography on the macroscale adhesion.

This collection of nine papers highlights just a small sample of recent developments in the broad and active field of tribology. Even with a limited sample size, though, several of the papers point at two common themes for the continued expansion of the field. First, the development of in-situ experimental tools with advanced spectroscopic and imaging methods (e.g., AFM-based infrared spectroscopy) will enable new insight into the chemistry, structure, and properties of tribological films at different time and length scales. Experimental Mechanics focuses on the advent of such experimental methods, and as such, will continue to be a valuable journal for pioneering research in tribology. Second, it has been shown that a complete understanding of tribological phenomena requires significant innovations at the borders between physics, chemistry, biology, mathematics, and engineering. To achieve these advancements, venues that bring together diverse experts and encourage multi-disciplinary research will be required, such as the Gordon Research Conference on Tribology, STLE Tribology Frontiers Conference, International Conference on BioTribology, and SEM International Symposium on Micro- and Nanomechanics. This special issue was only possible through the support of the past Experimental 
Mechanics Editor-in-Chief, Professor Ioannis Chasiotis, the current Experimental Mechanics Editor-in-Chief, Professor Alan Zehnder, and the Experimental Mechanics editorial team.
Publisher's Note Springer Nature remains neutral with regard to jurisdictional claims in published maps and institutional affiliations. 